\title{
SATURATIONS OF POWERS OF CERTAIN DETERMINANTAL IDEALS
}

\author{
KOSUKE FUKUMURO, TARO INAGAWA AND KOJI NISHIDA
}

ABSTRACT. In this paper we study certain determinantal ideals that extend the class of ideals of Herzog-Northcott type introduced by O'Carroll and Planas-Vilanova. As is well known, in a three-dimensional Cohen-Macaulay local ring, the second symbolic powers of ideals of HerzogNorthcott type can be controlled well. We aim to generalize this fact considering "saturation" instead of "symbolic power." Furthermore, in order to compare the saturation with the symbolic power, we study the associated primes of the powers of certain determinantal ideals.

1. Introduction. Let $R$ be a Noetherian ring and $m$ an integer with $m \geq 2$. Let $x_{1}, x_{2}, \ldots, x_{m+1}$ be a sequence of elements of $R$ generating a proper ideal of height $m+1$, and let $\left\{\alpha_{i j}\right\}$ be a family of positive integers, where $i=1,2, \ldots, m$ and $j=1,2, \ldots, m+1$. We set

$$
a_{i j}= \begin{cases}x_{i+j-1}^{\alpha_{i j}} & \text { if } i+j \leq m+2 \\ x_{i+j-m-2}^{\alpha_{i j}} & \text { if } i+j>m+2\end{cases}
$$

for any $i=1,2, \ldots, m$ and $j=1,2, \ldots, m+1$, and consider the matrix $A=\left(a_{i j}\right)$ of size $m \times(m+1)$. If $\alpha_{i j}=1$ for all $i$ and $j$, the matrix $A$ looks like

$$
\left(\begin{array}{ccccc}
x_{1} & x_{2} & \ldots & x_{m} & x_{m+1} \\
x_{2} & \ldots & x_{m} & x_{m+1} & x_{1} \\
\vdots & . \cdot & . \cdot & . \cdot & \vdots \\
x_{m} & x_{m+1} & x_{1} & \ldots & x_{m-1}
\end{array}\right) .
$$

However, we may put any exponents to each entry. In this paper, we study the ideal generated by the maximal minors of $A$. If $m=2$, this kind of ideal is known as Herzog-Northcott type in the recent literature

The third author is supported by KAKENHI (23540042). The third author is the corresponding author.

Received by the editors on July 25, 2013, and in revised form on March 18, 2014. DOI: $10.1216 /$ JCA-2015-7-2-167 
[18], and it is a well-known result of Herzog [12] that the defining ideal of a space monomial curves is Herzog-Northcott type. Because the ideals of Herzog-Northcott type provide interesting examples of symbolic Rees algebras, a lot of authors studied the symbolic powers of those ideals (cf., $[\mathbf{5}, \mathbf{8}, \mathbf{9}, \mathbf{1 1}, \mathbf{1 3}, \mathbf{1 6}, \mathbf{2 1}])$. Although the symbolic powers of ideals usually behave very wildly, if the ideal is HerzogNorthcott type, its second symbolic power can be controlled well (cf., $[10,15,17])$. The purpose of this paper is to generalize this fact for ideals stated above replacing "symbolic power" by "saturation." In order to explain our main result, let us recall the definitions of the symbolic power and the saturation of an ideal.

Let $(R, \mathfrak{m})$ be a local ring and $I$ an ideal of $R$ such that $\operatorname{dim} R / I>0$. Let $r$ be a positive integer. We set

$$
\left(I^{r}\right)^{\text {sat }}=\left\{x \in R \mid \mathfrak{m}^{i} \cdot x \subseteq I^{r} \text { for some integer } i \geq 0\right\}
$$

and call it the saturation of $I^{r}$. As $\left(I^{r}\right)^{\text {sat }} / I^{r} \cong \mathrm{H}_{\mathfrak{m}}^{0}\left(R / I^{r}\right)$, where $\mathrm{H}_{\mathfrak{m}}^{0}(\cdot)$ denotes the 0 -th local cohomology functor, we have $\left(I^{r}\right)^{\text {sat }}=I^{r}$ if and only if depth $R / I^{r}>0$. Moreover, if $J$ is an m-primary ideal such that depth $R /\left(I^{r}:_{R} J\right)>0$, we have $\left(I^{r}\right)^{\text {sat }}=I^{r}:_{R} J$. On the other hand, the $r$-th symbolic power of $I$ is defined by

$$
\begin{aligned}
I^{(r)}=\left\{x \in R \mid s x \in I^{r} \text { for some } s\right. & \in R \text { such that } \\
& \left.s \notin \mathfrak{p} \text { for any } \mathfrak{p} \in \operatorname{Min}_{R} R / I\right\} .
\end{aligned}
$$

In order to compare $\left(I^{r}\right)^{\text {sat }}$ and $I^{(r)}$, let us take a minimal primary decomposition of $I^{r}$;

$$
I^{r}=\bigcap_{\mathfrak{p} \in \operatorname{Ass}_{R} R / I^{r}} \mathrm{Q}(\mathfrak{p}),
$$

where $\mathrm{Q}(\mathfrak{p})$ denotes the $\mathfrak{p}$-primary component. It is easy to see that

$$
\left(I^{r}\right)^{\text {sat }}=\bigcap_{\mathfrak{m} \neq \mathfrak{p} \in \operatorname{Ass}_{R} R / I^{r}} \mathrm{Q}(\mathfrak{p}) \text { and } \quad I^{(r)}=\bigcap_{\mathfrak{p} \in \operatorname{Min}_{R} R / I} \mathrm{Q}(\mathfrak{p}) .
$$

Hence, we have $\left(I^{r}\right)^{\text {sat }} \subseteq I^{(r)}$ and the equality holds if and only if $\operatorname{Ass}_{R} R / I^{r}$ is a subset of $\{\mathfrak{m}\} \cup \operatorname{Min}_{R} R / I$. Therefore, if $\operatorname{dim} R / I=1$, then $\left(I^{r}\right)^{\text {sat }}=I^{(r)}$. If $\operatorname{dim} R / I \geq 2,\left(I^{r}\right)^{\text {sat }}$ may be different from $I^{(r)}$, but even in that case, $\left(I^{r}\right)^{\text {sat }}$ has meaning as an approximation of $I^{(r)}$. 
If $(R, \mathfrak{m})$ is a three-dimensional Cohen-Macaulay local ring and $I$ is an ideal of Herzog-Northcott type, then $I^{(2)} / I^{2}$ is a cyclic $R$-module and its generator can be described precisely (cf., [10, (2.2), (2.3)]). This fact can be generalized as follows, which is the main result of this paper.

Theorem 1.1. Let $(R, \mathfrak{m})$ be a Cohen-Macaulay local ring of $\operatorname{dim} R=$ $m+1$, where $m \geq 2$. Let $x_{1}, x_{2}, \ldots, x_{m+1}$ be an sop for $R$, and set $I$ to be the ideal generated by the maximal minors of $A$. Then the following assertions hold.

(1) $\left(I^{r}\right)^{\text {sat }}=I^{r}$ for any $r=1, \ldots, m-1$.

(2) $\left(I^{m}\right)^{\text {sat }} / I^{m}$ is a cyclic $R$-module.

The proof of this theorem is given in Section 4. Moreover, for $I$ of 1.1 , we can describe a generator of $\left(I^{m}\right)^{\text {sat }} / I^{m}$ assuming suitable conditions on $\left\{\alpha_{i j}\right\}$. In order to compare $\left(I^{r}\right)^{\text {sat }}$ with $I^{(r)}$ for $I$ of 1.1 , we have to compare $\operatorname{Ass}_{R} R / I^{r}$ with $\{\mathfrak{m}\} \cup \operatorname{Min}_{R} R / I$. For that purpose, we study the associated primes of powers of ideals in a more general situation in Section 3. Our results are closely related to [3, Lemma 3.3, Corollary 3.5] and the frameworks for the proofs are similar. Anyway, as a corollary of the results stated in Section 3, we see that the ideal $I$ of 1.1 satisfies $\left(I^{r}\right)^{\text {sat }} \subsetneq I^{(r)}$ if $r>m \geq 3$ and $\alpha_{i j}=1$ for any $i, j$.

Throughout this paper, $R$ is a commutative ring, and we often assume that $R$ is a Noetherian local ring with the maximal ideal $\mathfrak{m}$. For positive integers $m, n$ and an ideal $\mathfrak{a}$ of $R$, we denote by Mat $(m, n ; \mathfrak{a})$ the set of $m \times n$ matrices with entries in $\mathfrak{a}$. For any $A \in \operatorname{Mat}(m, n ; R)$ and any $k \in \mathbb{Z}$ we denote by $\mathrm{I}_{k}(A)$ the ideal generated by the $k$-minors of $A$. In particular, $\mathrm{I}_{k}(A)$ is defined to be $R$ (respectively, $\left.(0)\right)$ for $k \leq 0$ (respectively, $k>\min \{m, n\}$ ). If $A, B \in \operatorname{Mat}(m, n ; R)$ and the $(i, j)$ entries of $A$ and $B$ are congruent modulo a fixed ideal $\mathfrak{a}$ for any $(i, j)$, we write $A \equiv B \bmod \mathfrak{a}$.

2. Preliminaries. In this section, we assume that $R$ is just a commutative ring. Let $m, n$ be positive integers with $m \leq n$ and $A=\left(a_{i j}\right) \in \operatorname{Mat}(m, n ; R)$. Let us recall the following rather wellknown fact. 
Lemma 2.1. Suppose $\mathrm{I}_{m}(A) \subseteq \mathfrak{p} \in \operatorname{Spec} R$, and put $\ell=\max \{0 \leq k \in$ $\left.\mathbb{Z} \mid \mathrm{I}_{k}(A) \nsubseteq \mathfrak{p}\right\}$. Then $\ell<m$ and there exists $B \in \operatorname{Mat}\left(m-\ell, n-\ell ; \mathfrak{p} R_{\mathfrak{p}}\right)$ such that $\mathrm{I}_{k}(A)_{\mathfrak{p}}=\mathrm{I}_{k-\ell}(B)$ for any $k \in \mathbb{Z}$.

Proof. We prove by induction on $\ell$. The assertion is obvious if $\ell=0$. So, let us consider the case where $\ell>0$. Then $\mathrm{I}_{1}(A) \nsubseteq \mathbb{p}$, and so some entry of $A$ is a unit in $R_{\mathfrak{p}}$. Hence, applying elementary operations to $A$ in $\operatorname{Mat}\left(m, n ; R_{\mathfrak{p}}\right)$, we get a matrix of the form

$$
\left(\begin{array}{c|ccc}
1 & 0 & \cdots & 0 \\
\hline 0 & & & \\
\vdots & & & A^{\prime} \\
0 & & &
\end{array}\right),
$$

where $A^{\prime} \in \operatorname{Mat}\left(m-1, n-1 ; R_{\mathfrak{p}}\right)$. It is easy to see that $\mathrm{I}_{k}(A)_{\mathfrak{p}}=$ $\mathrm{I}_{k-1}\left(A^{\prime}\right)$ for any $k \in \mathbb{Z}$. Hence $\mathrm{I}_{m-1}\left(A^{\prime}\right) \subseteq \mathfrak{p} R_{\mathfrak{p}}$ and $\ell-1=\max \{0 \leq$ $\left.k \in \mathbb{Z} \mid \mathrm{I}_{k}\left(A^{\prime}\right) \nsubseteq \mathfrak{p} R_{\mathfrak{p}}\right\}$. By the induction hypothesis, there exists

$B \in \operatorname{Mat}\left((m-1)-(\ell-1),(n-1)-(\ell-1) ; \mathfrak{p} R_{\mathfrak{p}}\right)=\operatorname{Mat}\left(m-\ell, n-\ell ; \mathfrak{p} R_{\mathfrak{p}}\right)$

such that $\mathrm{I}_{t}\left(A^{\prime}\right)=\mathrm{I}_{t-(\ell-1)}(B)$ for any $t \in \mathbb{Z}$. Then we have $\mathrm{I}_{k}(A)_{\mathfrak{p}}=$ $\mathrm{I}_{k-\ell}(B)$ for any $k \in \mathbb{Z}$.

In the rest of this section, we assume $n=m+1$. For any $j=1,2, \ldots, m+1, A_{j}$ denotes the $m \times m$ submatrix of $A$ determined by removing the $j$-th column. We set $d_{j}=(-1)^{j-1} \cdot \operatorname{det} A_{j}$ and $I=\left(d_{1}, d_{2}, \ldots, d_{m+1}\right) R=\mathrm{I}_{m}(A)$. Let us take an indeterminate $t$ over $R$ and consider the Rees algebra of $I$;

$$
\mathscr{R}(I):=R\left[d_{1} t, d_{2} t, \ldots, d_{m+1} t\right] \subseteq R[t],
$$

which is a graded ring such that $\operatorname{deg} d_{j} t=1$ for all $j=1,2, \ldots, m+1$. On the other hand, let $S=R\left[T_{1}, T_{2}, \ldots, T_{m+1}\right]$ be a polynomial ring over $R$ with $m+1$ variables. We regard $S$ as a graded ring by setting $\operatorname{deg} T_{j}=1$ for all $j=1,2, \ldots, m+1$. Let $\pi: S \longrightarrow \mathscr{R}(I)$ be the homomorphism of $R$-algebras such that $\pi\left(T_{j}\right)=d_{j} t$ for any $j$. Then $\pi$ is a surjective graded homomorphism. Now we set

$$
f_{i}=\sum_{j=1}^{m+1} x_{i j} T_{j} \in S_{1}
$$


for any $i=1, \ldots, m$. It is easy to see $\left(f_{1}, f_{2}, \ldots, f_{m}\right) S \subseteq \operatorname{Ker} \pi$. For our purposes, the following result due to Avramov [2] is very important (another elementary proof is given in [6]).

Proposition 2.2. Suppose that $R$ is a Noetherian ring. If grade $\mathrm{I}_{k}(A) \geq$ $m-k+2$ for all $k=1, \ldots, m$, then $\operatorname{Ker} \pi=\left(f_{1}, f_{2}, \ldots, f_{m}\right) S$ and $f_{1}, f_{2}, \ldots, f_{m}$ is an $S$-regular sequence.

As the last preliminary result, we describe a technique using determinants of matrices. Suppose that $y_{1}, y_{2}, \ldots, y_{m+1}$ are elements of $R$ such that

$$
A\left(\begin{array}{c}
y_{1} \\
y_{2} \\
\vdots \\
y_{m+1}
\end{array}\right)=\left(\begin{array}{c}
0 \\
0 \\
\vdots \\
0
\end{array}\right) \text {. }
$$

We put $y=y_{1}+y_{2}+\cdots+y_{m+1}$.

Lemma 2.3. If $y, y_{k}$ form a regular sequence for some $k=1,2, \ldots, m+$ 1 , then there exists $\delta \in R$ such that $y_{j} \cdot \delta=d_{j}$ for any $j=1,2, \ldots, m+1$.

Proof. We put $d=d_{1}+d_{2}+\cdots+d_{m+1}$. Then the following assertion holds:

Claim. $y \cdot d_{j}=y_{j} \cdot d$ for all $j=1,2, \ldots, m+1$.

In order to prove the claim above, let us consider the following $(m+$ 1) $\times(m+1)$ matrix:

$$
B=\left(\begin{array}{cccc}
1 & 1 & \cdots & 1 \\
\hline & & A & \\
& &
\end{array}\right)
$$

Expanding $\operatorname{det} B$ along the first row, we get $\operatorname{det} B=d$. Let us fix 
$j=1,2, \ldots, m+1$. Multiplying the $j$-th column of $B$ by $y_{j}$, we get

$$
B^{\prime}=\left(\begin{array}{ccccc}
1 & \cdots & y_{j} & \cdots & 1 \\
a_{11} & \cdots & a_{1 j} y_{j} & \cdots & a_{1, m+1} \\
\vdots & & \vdots & & \vdots \\
a_{m 1} & \cdots & a_{m j} y_{j} & \cdots & a_{m, m+1}
\end{array}\right) .
$$

Then $\operatorname{det} B^{\prime}=y_{j} \cdot \operatorname{det} B=y_{j} \cdot d$. Next, for any $\ell \in\{1,2, \ldots, m+1\} \backslash\{j\}$, we add the $\ell$-th column of $B^{\prime}$ multiplied by $y_{\ell}$ to the $j$-th column, and get

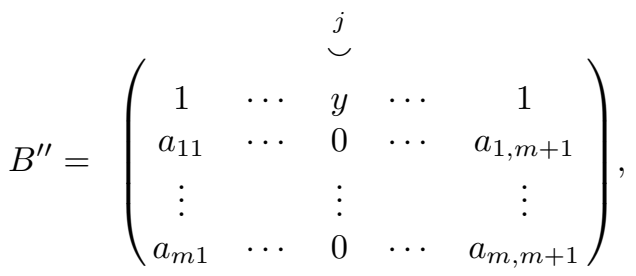

since our assumption means

$$
a_{i 1} y_{1}+\cdots+a_{i j} y_{j}+\cdots+a_{i, m+1} y_{m+1}=0
$$

for all $i=1, \ldots, m$. Then $\operatorname{det} B^{\prime \prime}=\operatorname{det} B^{\prime}=y_{j} \cdot d$. Finally, replacing the first $j$ columns of $B^{\prime \prime}$, we get

$$
B^{\prime \prime \prime}=\left(\begin{array}{c|ccc}
y & 1 & \cdots & 1 \\
\hline 0 & & & \\
\vdots & & A_{j} & \\
0 & & &
\end{array}\right) .
$$

Then $y \cdot d_{j}=y \cdot(-1)^{j-1} \cdot \operatorname{det} A_{j}=(-1)^{j-1} \cdot \operatorname{det} B^{\prime \prime \prime}=\operatorname{det} B^{\prime \prime}=y_{j} \cdot d$. Thus, we get the equalities of the claim.

Now we take $k=1,2, \ldots, m+1$ so that $y, y_{k}$ form a regular sequence. Because $y \cdot d_{k}=y_{k} \cdot d$, there exists $\delta \in R$ such that $d=y \delta$. Then $y \cdot d_{j}=y_{j} \cdot y \delta$ for any $j=1,2, \ldots, m+1$. As $y$ is an $R$-NZD, we get $d_{j}=y_{j} \cdot \delta$ for any $j=1,2, \ldots, m+1$, and the proof is complete.

Lemma 2.4. If $R$ is a Cohen-Macaulay local ring and $y_{1}, y_{2}, \ldots, y_{m+1}$ is an ssop for $R$, then $y, y_{k}$ form a regular sequence for any $k=$ $1,2, \ldots, m+1$. 
Proof. It is enough to show for $k=1$. Because $\left(y_{1}, y_{2}, \ldots, y_{m+1}\right) R=$ $\left(y, y_{1}, \ldots, y_{m}\right) R$, it follows that $y, y_{1}, \ldots, y_{m}$ is an ssop for $R$, too. Hence $y, y_{1}$ is $R$-regular.

Lemma 2.5. Suppose that $\mathfrak{a}$ is an ideal of $R$ and $a_{i j} \in \mathfrak{a}$ for all $i, j$. We put $Q=\left(y_{1}, y_{2}, \ldots, y_{m+1}\right) R$. Then $\delta$ of Lemma 2.3 is an element of $\mathfrak{a}^{m}:_{R} Q$.

Proof. We get this assertion since $d_{j} \in \mathfrak{a}^{m}$ for any $j=1,2, \ldots, m+1$.

3. Associated primes of $R / I^{n}$. Let $R$ be a Noetherian ring and $A=\left(a_{i j}\right) \in \operatorname{Mat}(m, m+1 ; R)$, where $1 \leq m \in \mathbb{Z}$. Let $I=\mathrm{I}_{m}(A)$. Throughout this section, we assume that $I$ is a proper ideal and grade $\mathrm{I}_{k}(A) \geq m-k+2$ for all $k=1, \ldots, m$. Let us keep the notation of Section 2.

Let $K_{\bullet}$ be the Koszul complex of $f_{1}, f_{2}, \ldots, f_{m}$, which is a complex of graded free $S$-modules. We denote its boundary map by $\partial_{\bullet}$. Let $e_{1}, e_{2}, \ldots, e_{m}$ be an $S$-basis of $K_{1}$ consisting of homogeneous elements of degree 1 such that $\partial_{1}\left(e_{i}\right)=f_{i}$ for any $i=1, \ldots, m$. Then, for any $s=1, \ldots, m$,

$$
\left\{e_{i_{1}} \wedge e_{i_{2}} \wedge \cdots \wedge e_{i_{s}} \mid 1 \leq i_{1}<i_{2}<\cdots<i_{s} \leq m\right\}
$$

is an $S$-basis of $K_{s}$ consisting of homogeneous elements of degree $s$, and we have

$$
\partial_{s}\left(e_{i_{1}} \wedge e_{i_{2}} \wedge \cdots \wedge e_{i_{s}}\right)=\sum_{p=1}^{s}(-1)^{p-1} \cdot f_{i_{p}} \cdot e_{i_{1}} \wedge \cdots \wedge \widehat{e_{i_{p}}} \wedge \cdots \wedge e_{i_{s}}
$$

where $\widehat{e_{i_{p}}}$ means that $e_{i_{p}}$ is omitted from the exterior product. Let $1 \leq r \in \mathbb{Z}$. Taking the homogeneous part of degree $r$ of $K_{\bullet}$, we get a complex

$$
\left[K_{\bullet}\right]_{r}: 0 \longrightarrow\left[K_{m}\right]_{r} \stackrel{\partial_{m}}{\longrightarrow}\left[K_{m-1}\right]_{r} \longrightarrow \cdots \longrightarrow\left[K_{1}\right]_{r} \stackrel{\partial_{1}}{\longrightarrow}\left[K_{0}\right]_{r} \longrightarrow 0
$$

of finitely generated free $R$-modules. It is obvious that $\left[K_{s}\right]_{r}=0$ if $r<s$. On the other hand, if $r \geq s$, then

$$
\left\{\begin{array}{l|l}
T_{1}^{\alpha_{1}} T_{2}^{\alpha_{2}} \cdots T_{m+1}^{\alpha_{m+1}} \cdot e_{i_{1}} \wedge e_{i_{2}} \wedge \cdots \wedge e_{i_{s}} & \begin{array}{l}
0 \leq \alpha_{1}, \alpha_{2}, \ldots, \alpha_{m+1} \in \mathbb{Z} \\
\alpha_{1}+\alpha_{2}+\cdots+\alpha_{m+1}=r-s \\
1 \leq i_{1}<i_{2}<\ldots<i_{s} \leq m
\end{array}
\end{array}\right\}
$$


is an $R$-basis of $\left[K_{s}\right]_{r}$.

Proposition 3.1. If $(R, \mathfrak{m})$ is a local ring and $A \in \operatorname{Mat}(m, m+1 ; \mathfrak{m})$, we have

$$
\text { proj. } \operatorname{dim}_{R} R / I^{r}= \begin{cases}r+1 & \text { if } r<m, \\ m+1 & \text { if } r \geq m .\end{cases}
$$

Proof. By Proposition 2.2 and $[4,1.6 .17]$, we see that

$$
0 \longrightarrow K_{m} \stackrel{\partial_{m}}{\longrightarrow} K_{m-1} \longrightarrow \cdots \longrightarrow K_{1} \stackrel{\partial_{1}}{\longrightarrow} K_{0} \stackrel{\pi}{\longrightarrow} \mathscr{R}(I) \longrightarrow 0
$$

is a graded $S$-free resolution of $\mathscr{R}(I)$. Hence, for any integer $r \geq 0$,

$$
0 \longrightarrow\left[K_{m}\right]_{r} \stackrel{\partial_{m}}{\longrightarrow}\left[K_{m-1}\right]_{r} \longrightarrow \cdots \longrightarrow\left[K_{1}\right]_{r} \stackrel{\partial_{1}}{\longrightarrow}\left[K_{0}\right]_{r} \stackrel{\pi}{\longrightarrow} I^{r} t^{r} \longrightarrow 0
$$

is an $R$-free resolution of the $R$-module $I^{r} t^{r}$. Let us notice $I^{r} t^{r} \cong I^{r}$ as $R$-modules. Suppose $1 \leq s \leq m$ and $r \geq s$. Then, for any non-negative integers $\alpha_{1}, \alpha_{2}, \ldots, \alpha_{m+1}$ with $\alpha_{1}+\alpha_{2}+\cdots+\alpha_{m+1}=r-s$ and positive integers $i_{1}, i_{2}, \ldots, i_{s}$ with $1 \leq i_{1}<i_{2}<\cdots<i_{\ell} \leq m$, we have

$$
\begin{aligned}
\partial_{s} & \left(T_{1}^{\alpha_{1}} T_{2}^{\alpha_{2}} \cdots T_{m+1}^{\alpha_{m+1}} \cdot e_{i_{1}} \wedge e_{i_{2}} \wedge \cdots \wedge e_{i_{s}}\right) \\
= & T_{1}^{\alpha_{1}} T_{2}^{\alpha_{2}} \cdots T_{m+1}^{\alpha_{m+1}} \cdot \sum_{p=1}^{s}(-1)^{p-1} \\
& \cdot\left(\sum_{j=1}^{m+1} a_{i_{p}, j} T_{j}\right) \cdot e_{i_{1}} \wedge \cdots \wedge \widehat{e_{i_{p}}} \wedge \cdots \wedge e_{i_{s}} \\
= & \sum_{p=1}^{s} \sum_{j=1}^{m+1}(-1)^{p-1} a_{i_{p}, j} \cdot T_{1}^{\alpha_{1}} \cdots T_{j}^{1+\alpha_{j}} \cdots T_{m+1}^{\alpha_{m+1}} \cdot e_{i_{1}} \\
& \wedge \cdots \wedge \widehat{e_{i_{p}}} \wedge \cdots \wedge e_{i_{s}} \\
\in & \mathfrak{m} \cdot\left[K_{s-1}\right]_{r} .
\end{aligned}
$$

Hence, $\left[K_{\bullet}\right]_{r}$ gives a minimal $R$-free resolution of $I^{r}$. If $r<m$, we have $\left[K_{r}\right]_{r} \neq 0$ and $\left[K_{s}\right]_{r}=0$ for any $s>r$, and so proj. $\operatorname{dim}_{R} I^{r}=r$. On the other hand, if $r \geq m$, we have $\left[K_{m}\right]_{r} \neq 0$ and $\left[K_{s}\right]_{r}=0$ for any $s>m$, and so proj. $\operatorname{dim}_{R} I^{r}=m$. Thus, we get the required equality as proj. $\operatorname{dim}_{R} R / I^{r}=$ proj. $\operatorname{dim}_{R} I^{r}+1$. 
By the Auslander-Buchsbaum formula (cf., [4, 1.3.3]), we get the following.

Corollary 3.2. If $(R, \mathfrak{m})$ is local and $A \in \operatorname{Mat}(m, m+1 ; \mathfrak{m})$, we have $\operatorname{depth} R / I^{r}= \begin{cases}\operatorname{depth} R-r-1 & \text { if } r<m, \\ \operatorname{depth} R-m-1 & \text { if } r \geq m .\end{cases}$

Here we remark that $\operatorname{depth} R \geq \operatorname{grade}_{1}(A) \geq m+1$ by our assumption of this section. As a consequence of Corollary 3.2, we see that the next assertion holds.

Corollary 3.3. Suppose that $(R, \mathfrak{m})$ is a local ring and $A \in \operatorname{Mat}(m, m+$ $1 ; \mathfrak{m})$. Then we have $\mathfrak{m} \in \operatorname{Ass}_{R} R / I^{r}$ if and only if $r \geq m$ and depth $R=m+1$.

The next result is a generalization of Corollary 3.3.

Proposition 3.4. Let $I \subseteq \mathfrak{p} \in \operatorname{Spec} R$ and $1 \leq r \in \mathbb{Z}$. We put

$$
\ell=\max \left\{0 \leq k<m \mid \mathrm{I}_{k}(A) \not \mathbb{p}\right\} .
$$

Then the following conditions are equivalent.

(1) $\mathfrak{p} \in \operatorname{Ass}_{R} R / I^{r}$.

(2) $r \geq m-\ell$ and depth $R_{\mathfrak{p}}=m-\ell+1$.

When this is the case, grade $\mathrm{I}_{\ell+1}(A)=m-\ell+1$.

Proof. By Lemma 2.1, there exists $B \in \operatorname{Mat}\left(m-\ell, m-\ell+1 ; \mathfrak{p} R_{\mathfrak{p}}\right)$ such that $\mathrm{I}_{k}(B)=\mathrm{I}_{k+\ell}(A)_{\mathfrak{p}}$ for any $k \in \mathbb{Z}$. Hence, for any $k=$ $1, \ldots, m-\ell$, we have

$$
\operatorname{grade} \mathrm{I}_{k}(B)=\operatorname{grade} \mathrm{I}_{k+\ell}(A)_{\mathfrak{p}} \geq m-(k+\ell)+2=(m-\ell)-k+2 .
$$

Therefore, by Corollary 3.3, we see that $\mathfrak{p} R_{\mathfrak{p}} \in \operatorname{Ass}_{R_{\mathfrak{p}}} R_{\mathfrak{p}} / \mathrm{I}_{m-\ell}(B)^{r}$ if and only if $r \geq m-\ell$ and $\operatorname{depth} R_{\mathfrak{p}}=m-\ell+1$. Let us notice $\mathrm{I}_{m-\ell}(B)=I_{\mathfrak{p}}$. Because $\mathfrak{p} \in \operatorname{Ass}_{R} R / I^{r}$ if and only if $\mathfrak{p} R_{\mathfrak{p}} \in$ $\operatorname{Ass}_{R_{\mathfrak{p}}} R_{\mathfrak{p}} / I_{\mathfrak{p}}{ }^{r}$, we see $(1) \Leftrightarrow(2)$. Furthermore, as $\mathrm{I}_{\ell+1}(A) \subseteq \mathfrak{p}$, we have grade $\mathrm{I}_{\ell+1}(A) \leq \operatorname{depth} R_{\mathfrak{p}}$, and so we get grade $\mathrm{I}_{\ell+1}(A)=m-\ell+1$ if condition (2) is satisfied. 
For any positive integer $r$, let $\Lambda_{A}^{r}$ be the set of integers $i$ such that $\max \{1, m-r+1\} \leq i \leq m$ and $\operatorname{grade}_{i}(A)=m-i+2$. We denote by $\operatorname{Assh}_{R} R / \mathrm{I}_{i}(A)$ the set of $\mathfrak{p} \in \operatorname{Ass}_{R} R / \mathrm{I}_{i}(A)$ such that $\operatorname{dim} R / \mathfrak{p}=\operatorname{dim} R / \mathrm{I}_{i}(A)$. Then the following assertion holds.

Proposition 3.5. Let $R$ be a Cohen-Macaulay ring. Then, for any positive integer $r$, we have

$$
\operatorname{Ass}_{R} R / I^{r}=\bigcup_{i \in \Lambda_{A}^{r}} \operatorname{Assh}_{R} R / \mathrm{I}_{i}(A) .
$$

Proof. Let us take any $\mathfrak{p} \in \operatorname{Ass}_{R} R / I^{r}$ and put $\ell=\max \{0 \leq k<$ $\left.m \mid \mathrm{I}_{k}(A) \nsubseteq \mathfrak{p}\right\}$. Then $\mathrm{I}_{\ell+1}(A) \subseteq \mathfrak{p}$. Moreover, by Proposition 3.4, we have $r \geq m-\ell$, depth $R_{\mathfrak{p}}=m-\ell+1$ and $\operatorname{grade} \mathrm{I}_{\ell+1}(A)=m-\ell+1$. Hence $\ell+1 \in \Lambda_{A}^{r}$. Let us notice that ht $\mathfrak{p}=\operatorname{depth} R_{\mathfrak{p}}$ and ht $\mathrm{I}_{\ell+1}(A)=$ grade $\mathrm{I}_{\ell+1}(A)$ as $R$ is Cohen-Macaulay. Therefore ht $\mathfrak{p}=$ ht $\mathrm{I}_{\ell+1}(A)$, which means $\mathfrak{p} \in \operatorname{Assh}_{R} R / \mathrm{I}_{\ell+1}(A)$.

Conversely, let us take any $i \in \Lambda_{A}^{r}$ and $\mathfrak{q} \in \operatorname{Assh}_{R} R / \mathrm{I}_{i}(M)$. Then ht $\mathfrak{q}=$ ht $\mathrm{I}_{i}(A)=$ grade $\mathrm{I}_{i}(A)=m-i+2$. As our assumption implies ht $\mathrm{I}_{i-1}(A) \geq m-i+3$, it follows that $i-1=\max \{0 \leq k<m$ $\left.\mathrm{I}_{k}(A) \nsubseteq \mathfrak{q}\right\}$. Let us notice $r \geq m-(i-1)$ as $m-r+1 \leq i$, which is one of the conditions for $i \in \Lambda_{A}^{r}$. Moreover, we have $\operatorname{depth} R_{\mathfrak{q}}=$ ht $\mathfrak{q}=m-(i-1)+1$. Thus we get $\mathfrak{q} \in \operatorname{Ass}_{R} R / I^{r}$ by Proposition 3.4, and the proof is complete.

As a natural question, one may ask whether the results stated above can be extended to the case where $A=\left(a_{i j}\right) \in \operatorname{Mat}(m, n ; R)$ with $m<n$. As far as the authors know, the following two kinds of generalizations seem to be possible.

First, we would like to suggest considering the powers of modules. We set $M$ to be the cokernel of the $R$-linear map $R^{m} \longrightarrow R^{n}$ defined by ${ }^{t} A$. Let us assume grade $\mathrm{I}_{k}(A) \geq m-k+2$ for all $k=1, \ldots, m$. Then, by [2, Proposition 4], $M$ has rank $n-m$ and the $r$-th symmetric power $\mathscr{S}^{r}(M)$ is torsion-free over $R$ for any $r \geq 1$. In this case, $M$ can be embedded into a finitely generated free $R$-module $F$ and the $r$-th power $M^{r}$ of $M$ is defined to be the image of $\mathscr{S}^{r}(M)$ in $\mathscr{S}^{r}(F)$. Similarly as the case of $m \times(m+1)$ matrix, we consider the polynomial $\operatorname{ring} S=R\left[T_{1}, T_{2}, \ldots, T_{n}\right]$ and set $f_{i}=a_{i 1} T_{1}+a_{i 2} T_{2}+\cdots+a_{i n} T_{n} \in S$ for all $i=1, \ldots, m$. Then, by [2, Propsosition 1 and Proposition 4], we 
get an $R$-free resolution of $\mathscr{S}^{r}(F) / M^{r}$ by taking the homogeneous part of the Koszul complex of $f_{1}, f_{2}, \ldots, f_{m}$ over $S$. In this way, detailed information on the associated primes of $\mathscr{S}^{r}(F) / M^{r}$ could be deduced.

On the other hand, by using the free resolution of Akin-BuchsbaumWeyman [1], another generalization seems to be possible. This idea was suggested by the referee. Similarly as in the case of the $m \times(m+1)$ matrix, we set $I$ to be the ideal generated by the maximal minors of $A$,. Let us assume grade $\mathrm{I}_{k}(A) \geq(m-k+1)(n-m)+1$ for any $k=1, \ldots, m$. Then, by [1, Theorem 5.4], we get an $R$-free resolution of $R / I^{r}$ for any $r \geq 1$, and it could be used in place of Proposition 3.1 to deduce the $m \times n$ matrix version of Propositions 3.4 and 3.5.

Proposition 3.6. Let $R$ be an $(m+1)$-dimensional Cohen-Macaulay local ring, where $2 \leq m \in \mathbb{Z}$. Let $A$ be the matrix given in the introduction. Then the following assertions hold:

(1) ht $\mathrm{I}_{k}(A) \geq m-k+2$ for all $k=1,2, \ldots, m$.

(2) proj. $\operatorname{dim}_{R} R / I^{r}= \begin{cases}r+1 & \text { if } r<m, \\ m+1 & \text { if } r \geq m \text {. }\end{cases}$

(3) $\operatorname{depth} R / I^{r}= \begin{cases}m-r & \text { if } r<m, \\ 0 & \text { if } r \geq m .\end{cases}$

Furthermore, if $\alpha_{i j}=1$ for all $i$ and $j$, the following assertions hold:

(4) ht $\mathrm{I}_{2}(A)=m$ and $\operatorname{Assh}_{R} R / \mathrm{I}_{2}(A) \subseteq \operatorname{Ass}_{R} R / I^{r}$ for any $r \geq$ $m-1$.

(5) If $m$ is an odd integer with $m \geq 3$, then ht $\mathrm{I}_{3}(A)=m-1$ and $\operatorname{Assh}_{R} R / \mathrm{I}_{3}(A) \subseteq \operatorname{Ass}_{R} R / I^{r}$ for any $r \geq m-2$.

(6) If $m \geq 3$, then $\left(I^{r}\right)^{\text {sat }} \subsetneq I^{(r)}$ for any $r \geq m-1$.

Proof. (1) We aim to prove the following.

Claim. $\quad J_{k-1}+\mathrm{I}_{k}(A)$ is an $\mathfrak{m}$-primary ideal for any $k=1,2, \ldots, m$, where $J_{k-1}=\left(x_{1}, x_{2}, \ldots, x_{k-1}\right) R$.

If this is true, we have $\operatorname{dim} R / \mathrm{I}_{k}(A) \leq k-1$, and so ht $\mathrm{I}_{k}(A) \geq$ $\operatorname{dim} R-(k-1)=m-k+2$, which is the required inequality. 
In order to prove the claim, we take any $\mathfrak{p} \in \operatorname{Spec} R$ containing $J_{k-1}+\mathrm{I}_{k}(A)$. It is enough to show $J_{m+1}=\left(x_{1}, x_{2}, \ldots, x_{m+1}\right) R \subseteq \mathfrak{p}$. For that purpose, we prove $J_{\ell} \subseteq \mathfrak{p}$ for any $\ell=k-1, k, \ldots, m+1$ by induction on $\ell$. As we obviously have $J_{k-1} \subseteq \mathfrak{p}$, let us assume $k \leq \ell \leq m+1$ and $J_{\ell-1} \subseteq \mathfrak{p}$. Because the $k$-minor of $A$ with respect to the first $k$ rows and the columns $\ell-k+1, \ldots, \ell-1, \ell$ is congruent with

$$
\operatorname{det}\left(\begin{array}{rrrr}
0 & & x_{\ell, \ell-1}^{\alpha_{2,1}} & x_{\ell}^{\alpha_{1, \ell}} \\
& . & *
\end{array}\right)
$$

$\bmod J_{\ell-1}$, it follows that $J_{\ell-1}+\mathrm{I}_{k}(A)$ includes some power of $x_{\ell}$. Hence $x_{\ell} \in \mathfrak{p}$, and so we get $J_{\ell} \subseteq \mathfrak{p}$.

(2) and (3) follow from Proposition 3.1 and Corollary 3.2, respectively.

In the rest of this proof, we assume $\alpha_{i j}=1$ for any $i$ and $j$.

(4) Let $\mathfrak{q}=\left(x_{1}-x_{2}, x_{2}-x_{3}, \ldots, x_{m}-x_{m+1}\right) R$. Then $x_{1} \equiv x_{j} \bmod$ $\mathfrak{q}$ for any $j=1,2, \ldots, m+1$. Hence, any 2-minor of $A$ is congruent with

$$
\operatorname{det}\left(\begin{array}{ll}
x_{1} & x_{1} \\
x_{1} & x_{1}
\end{array}\right)=0
$$

$\bmod \mathfrak{q}$. This means $\mathrm{I}_{2}(A) \subseteq \mathfrak{q}$, and so ht $\mathrm{I}_{2}(A) \leq \mu_{R}(\mathfrak{q})=m$. On the other hand, ht $\mathrm{I}_{2}(A) \geq m$ by (1). Thus, we get ht $\mathrm{I}_{2}(A)=m$. Then, for

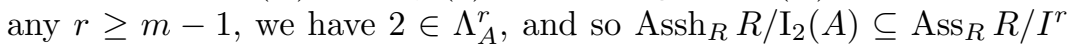
by Proposition 3.5.

(5) Let $\mathfrak{p}$ be the ideal of $R$ generated by $\left\{x_{i}-x_{i+2}\right\}$, where $i$ runs all odd integers with $1 \leq i \leq m-2$. Similarly, we set $\mathfrak{q}$ to be the ideal of $R$ generated by $\left\{x_{j}-x_{j+2}\right\}$, where $j$ runs all even integers with $2 \leq j \leq m-1$. Let $A^{\prime}$ be the submatrix of $A$ with the rows $i_{1}, i_{2}, i_{3}$ and the columns $j_{1}, j_{2}, j_{3}$, where $1 \leq i_{1}<i_{2}<i_{3} \leq m$ and $1 \leq j_{1}<j_{2}<j_{3} \leq m+1$. We can choose $p, q$ with $1 \leq p<q \leq 3$ so that $i_{p} \equiv i_{q}$ mod 2. Then, for any $t=1,2,3$, we have $i_{p}+j_{t} \equiv i_{q}+j_{t}$ $\bmod 2$, and so, if $i_{p}+j_{t}$ is odd (respectively, even), it follows that $a_{i_{p}, j_{t}} \equiv a_{i_{q}, j_{t}}$ mod $\mathfrak{q}$ (respectively, $\mathfrak{p}$ ). Hence, we see that the $p$-th row of $A^{\prime}$ is congruent with the $q$-th row of $A^{\prime} \bmod \mathfrak{p}+\mathfrak{q}$, which means $\operatorname{det} A^{\prime} \equiv 0 \bmod \mathfrak{p}+\mathfrak{q} . \quad$ As a consequence, we get $\mathrm{I}_{3}(A) \subseteq \mathfrak{p}+\mathfrak{q}$. 
Therefore, ht $\mathrm{I}_{3}(A) \leq \mu_{R}(\mathfrak{p})+\mu_{R}(\mathfrak{q})=(m-1) / 2+(m-1) / 2=m-1$.

(6) Let us take any $\mathfrak{p} \in \operatorname{Assh}_{R} R / \mathrm{I}_{2}(A)$ and $r \geq m-1$. Then, by (4) we have ht $\mathfrak{p}=m \geq 3$ and $\mathfrak{p} \in \operatorname{Ass}_{R} R / I^{r}$. Hence $\operatorname{Ass}_{R} R / I^{r}$ is not a subset of $\{\mathfrak{m}\} \cup \operatorname{Min}_{R} R / I$. Therefore, by the observation stated in the introduction, we get $\left(I^{r}\right)^{\text {sat }} \subsetneq I^{(r)}$, and the proof is complete.

4. Computing $\left(I^{m}\right)^{\text {sat }}$. In this section, we assume that $(R, \mathfrak{m})$ is an $(m+1)$-dimensional Cohen-Macaulay local ring, where $2 \leq m \in \mathbb{Z}$. Let $x_{1}, x_{2}, \ldots, x_{m+1}$ be an sop for $R$, and let $A$ be the matrix given in the introduction. We put $I=\mathrm{I}_{m}(A)$. Then, by (3) of Proposition 3.6, we get assertion (1) of Theorem 1.1. Let us prove Theorem 1.1 (2).

For any $j=1,2, \ldots, m+1$, we set $d_{j}=(-1)^{j-1} \cdot \operatorname{det} A_{j}$, where $A_{j}$ is the submatrix of $A$ determined by removing the $j$-th column. Then $I=\left(d_{1}, d_{2}, \ldots, d_{m+1}\right) R$. Furthermore, for any $k=1,2, \ldots, m+1$, we denote by $\beta_{k}$ the minimum of the exponents of $x_{k}$ that appear in the entries of $A$. Let us notice that $A$ 's entries which are powers of $x_{k}$ appear as follows:

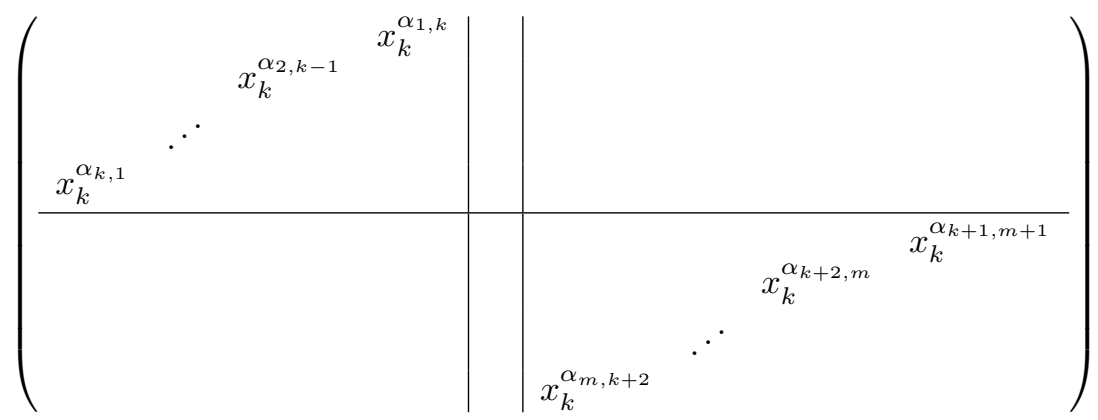

if $1 \leq k<m$, and

$$
\left(\begin{array}{ccc} 
& & x_{m}^{\alpha_{1, m}} \\
& x_{m}^{\alpha_{2, m-1}} & \\
x_{m}^{\alpha_{m, 1}} & &
\end{array}\right)
$$


or

$$
\left(\begin{array}{llll} 
& & & x_{m+1}^{\alpha_{1, m+1}} \\
& & x_{m+1}^{\alpha_{2, m}} & \\
& . & & \\
x_{m+1}^{\alpha_{m, 2}} & & &
\end{array}\right)
$$

if $k=m$ or $m+1$, respectively. So, we have

$$
\beta_{k}=\left\{\begin{array}{l}
\min \left\{\alpha_{i, k-i+1}\right\}_{1 \leq i \leq k} \cup\left\{\alpha_{i, k-i+m+2}\right\}_{k<i \leq m} \quad \text { if } 1 \leq k<m, \\
\min \left\{\alpha_{i, k-i+1}\right\}_{1 \leq i \leq m} \text { if } k=m \text { or } m+1
\end{array}\right.
$$

Then, for any $k=1,2, \ldots, m+1$, we can choose $i_{k}$ so that one of the following conditions is satisfied:

$$
\text { (i) } 1 \leq i_{k} \leq k \text { and } \beta_{k}=\alpha_{i_{k}, k-i_{k}+1}
$$

or

$$
\text { (ii) } k<i_{k} \leq m \text { and } \beta_{k}=\alpha_{i_{k}, k-i_{k}+m+2} \text {. }
$$

Now, for any $i=1,2, \ldots, m$ and $j=1,2, \ldots, m+1$, we set

$$
a_{i k}^{\prime}= \begin{cases}x_{k}^{\alpha_{i, k-i+1}-\beta_{k}} & \text { if } i \leq k \\ x_{k}^{\alpha_{i, k-i+m+2}-\beta_{k}} & \text { if } i>k .\end{cases}
$$

Then $a_{i_{k}, k}^{\prime}=1$ for all $k=1,2, \ldots, m+1$. The next assertion can be verified easily.

Lemma 4.1. Suppose $1 \leq i \leq m$ and $1 \leq j \leq m+1$.

(1) If $i+j \leq m+2$, setting $k=i+j-1$, we have $1 \leq k \leq m+1$, $i \leq k$ and $a_{i j}=x_{k}^{\beta_{k}} \cdot a_{i k}^{\prime}$.

(2) If $i+j>m+2$, setting $k=i+j-m-2$, we have $1 \leq k<m$, $i>k$ and $a_{i j}=x_{k}^{\beta_{k}} \cdot a_{i k}^{\prime}$.

Let $Q$ be the ideal of $R$ generated by $x_{1}^{\beta_{1}}, x_{2}^{\beta_{2}}, \ldots, x_{m+1}^{\beta_{m+1}}$. Then $A \in \operatorname{Mat}(m, m+1 ; Q)$ by Lemma 4.1. Assertion (2) of Theorem 1.1 follows from the next

Proposition 4.2. $\left(I^{m}\right)^{\mathrm{sat}}=I^{m}:_{R} Q$ and $\left(I^{m}\right)^{\text {sat }} / I^{m} \cong R / Q$. 
Proof. Let $S$ be the polynomial ring over $R$ with variables $T_{1}, T_{2}, \ldots$, $T_{m+1}$. We regard $S$ as a graded ring by setting $\operatorname{deg} T_{j}=1$ for any $j=1,2, \ldots, m+1$. Let

$$
f_{i}=\sum_{j=1}^{m+1} a_{i j} T_{j} \in S_{1}
$$

for any $i=1,2, \ldots, m$, and let $K_{\bullet}$ be the Koszul complex of $f_{1}, f_{2}, \ldots, f_{m}$. Then $K_{\bullet}$ is a graded complex. Let $\partial_{\bullet}$ be the boundary map of $K_{\bullet}$, and let $e_{1}, e_{2}, \ldots, e_{m}$ be an $S$-basis of $K_{1}$ consisting of homogeneous elements of degree 1 such that $\partial_{1}\left(e_{i}\right)=f_{i}$ for all $i=1,2, \ldots, m$. As is stated in the proof of Proposition 3.1,

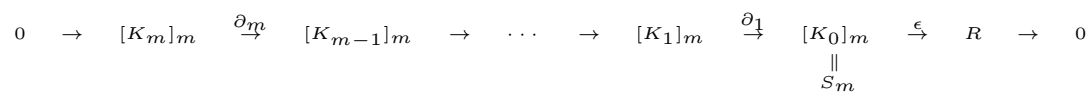

is an acyclic complex, where $\epsilon$ is the $R$-linear map such that

$$
\epsilon\left(T_{1}^{\alpha_{1}} T_{2}^{\alpha_{2}} \cdots T_{m+1}^{\alpha_{m+1}}\right)=d_{1}^{\alpha_{1}} d_{2}^{\alpha_{2}} \cdots d_{m+1}^{\alpha_{m+1}}
$$

for any non-negative integers $\alpha_{1}, \alpha_{2}, \ldots, \alpha_{m+1}$ with $\alpha_{1}+\alpha_{2}+\cdots+$ $\alpha_{m+1}=m$. We obviously have $\operatorname{Im} \epsilon=I^{m}$. We set $e=e_{1} \wedge e_{2} \wedge \cdots \wedge e_{m}$ and $\check{e}_{i}=e_{1} \wedge \cdots \wedge \widehat{e}_{i} \wedge \cdots \wedge e_{m}$ for any $i=1,2, \ldots, m$. Let us take $\{e\}$ and $\left\{T_{j} \check{e}_{i} \mid 1 \leq i \leq m, 1 \leq j \leq m+1\right\}$ as $R$-bases of $\left[K_{m}\right]_{m}$ and $\left[K_{m-1}\right]_{m}$, respectively. Because

$$
\partial_{m}(e)=\sum_{i=1}^{m}(-1)^{i-1} f_{i} \cdot \check{e}_{i}=\sum_{i=1}^{m} \sum_{j=1}^{m+1}(-1)^{i-1} a_{i j} \cdot T_{j} \check{e}_{i},
$$

we have $\partial_{m}\left(\left[K_{m}\right]_{m}\right) \subseteq Q \cdot\left[K_{m-1}\right]_{m}$. Hence, by $[\mathbf{1 4}, 3.1]$ we get

$$
\left(I^{m}:_{R} Q\right) / I^{m} \cong\left[K_{m}\right]_{m} / Q\left[K_{m}\right]_{m} \cong R / Q
$$

Here, for any $i=1,2, \ldots, m$ and $j=1,2, \ldots, m+1$, we set

$$
T_{i k}= \begin{cases}T_{k-i+1} & \text { if } i \leq k \\ T_{k-i+m+2} & \text { if } i>k\end{cases}
$$

Then the following assertion holds:

Claim 1. Suppose $1 \leq k, \ell \leq m+1$ and $k \neq \ell$. Then $T_{i k} \neq T_{i \ell}$ for any $i=1,2, \ldots, m$. 
In order to prove the above claim, we may assume $k<\ell$. Then the following three cases can happen: (i) $i \leq k<\ell$, (ii) $k<i \leq \ell$ or (iii) $k<\ell<i$. Because $k-i+1<\ell-i+1$ and $k-i+m+2<\ell-i+m+2$, we get $T_{i k} \neq T_{i \ell}$ in cases (i) and (iii). Furthermore, as $m+1>\ell-k$, we get $k-i+m+2>\ell-i+1$, and so $T_{i k} \neq T_{i \ell}$ holds also in the case of (ii). Thus, we have seen Claim 1.

Now, for any $k=1,2, \ldots, m+1$, we set

$$
v_{(k, e)}=\sum_{i=1}^{m}(-1)^{i-1} a_{i k}^{\prime} \cdot T_{i k} \check{e}_{i} \in\left[K_{m-1}\right]_{m} .
$$

Then the following equality holds:

Claim 2. $\partial_{m}(e)=\sum_{k=1}^{m+1} x_{k}^{\beta_{k}} \cdot v_{(k, e)}$.

In fact, by $(\sharp)$ and Lemma 4.1 , we have

$$
\begin{aligned}
& \partial_{m}(e)=\sum_{i=1}^{m}\left(\sum_{j=1}^{m-i+2}(-1)^{i-1} a_{i j} \cdot T_{j} \check{e}_{i}+\sum_{j=m-i+3}^{m+1}(-1)^{i-1} a_{i j} \cdot T_{j} \check{e}_{i}\right) \\
& =\sum_{i=1}^{m}\left(\sum_{k=i}^{m+1}(-1)^{i-1} x_{k}^{\beta_{k}} a_{i k}^{\prime} \cdot T_{k-i+1} \check{e}_{i}+\sum_{k=1}^{i-1}(-1)^{i-1} x_{k}^{\beta_{k}} a_{i k}^{\prime}\right. \\
& \left.\cdot T_{k-i+m+2} \check{e}_{i}\right) \\
& =\sum_{i=1}^{m} \sum_{k=1}^{m+1}(-1)^{i-1} x_{k}^{\beta_{k}} a_{i k}^{\prime} \cdot T_{i k} \check{e}_{i},
\end{aligned}
$$

and so the equality of Claim 2 follows.

Finally, we need the following:

Claim 3. $\left\{v_{(k, e)}\right\}_{1 \leq k \leq m}$ is a part of an $R$-basis of $\left[K_{m-1}\right]_{m}$.

If this is true, by $[\mathbf{1 4}, 3.4]$ (see $[\mathbf{7}, 3.4]$ for the case where $m=2$ ) we get $\operatorname{depth} R /\left(I^{m}:_{R} Q\right)>0$, which means $\left(I^{m}\right)^{\text {sat }}=I^{m}:_{R} Q$. So, let us prove Claim 3. By Claim 1, we see that $T_{i_{1}, 1} \check{e}_{i_{1}}, T_{i_{2}, 2} \check{e}_{i_{2}}, \ldots, T_{i_{m}, m} \check{e}_{i_{m}}$ 
are different to each other. We set

$$
U=\left\{T_{j} \check{e}_{i} \mid 1 \leq i \leq m, 1 \leq j \leq m+1\right\} \backslash\left\{T_{i_{k}, k} \check{e}_{i_{k}} \mid 1 \leq k \leq m\right\}
$$

and aim to prove that $U \cup\left\{v_{(k, e)}\right\}_{1 \leq k \leq m}$ is an $R$-basis of $\left[K_{m-1}\right]_{m}$. By $[14,3.3]$, it is enough to show that the submodule of $\left[K_{m-1}\right]_{m}$ generated by $U \cup\left\{v_{(k, e)}\right\}_{1 \leq k \leq m}$ includes $T_{i_{k}, k} \check{e}_{i_{k}}$ for any $k=1,2, \ldots, m$. This can be easily seen since

$$
v_{(k, e)}=(-1)^{k-1} \cdot T_{i_{k}, k} \check{e}_{i_{k}}+\sum_{i \neq i_{k}}(-1)^{i-1} a_{i k}^{\prime} \cdot T_{i k} \check{e}_{i}
$$

and $T_{i k} \check{e}_{i} \in U$ if $i \neq i_{k}$, which follows from Claim 1. Thus the assertion of Claim 3 follows, and the proof of Proposition 4.2 is complete.

If we assume a suitable condition on $\left\{\alpha_{i j}\right\}$, we can describe a generator of $\left(I^{m}\right)^{\text {sat }} / I^{m}$. For any $i=1,2, \ldots, m$ and $k=1,2, \ldots, m+1$, we set

$$
b_{i k}= \begin{cases}a_{i k}^{\prime} d_{k-i+1} & \text { if } i \leq k \\ a_{i k}^{\prime} d_{k-i+m+2} & \text { if } i>k\end{cases}
$$

and $B=\left(b_{i k}\right) \in \operatorname{Mat}(m, m+1 ; I)$. Then the next equality holds:

Lemma 4.3. $B\left(\begin{array}{c}x_{1}^{\beta_{1}} \\ x_{2}^{\beta_{2}} \\ \vdots \\ x_{m+1}^{\beta_{m+1}}\end{array}\right)=\left(\begin{array}{c}0 \\ 0 \\ \vdots \\ 0\end{array}\right)$.

Proof. For all $i=1,2, \ldots, m$, we have

$$
\sum_{j=1}^{m+1} a_{i j} d_{j}=0
$$

Let us divide the left side of this equality as follows:

$$
\sum_{j=1}^{m-i+2} a_{i j} d_{j}+\sum_{j=m-i+3}^{m+1} a_{i j} d_{j}=0
$$

If $1 \leq j \leq m-i+2$, setting $k=i+j-1$, we have $i \leq k \leq m+1$ and

$$
a_{i j} d_{j}=x_{k}^{\beta_{k}} a_{i k}^{\prime} \cdot d_{k-i+1}=x_{k}^{\beta_{k}} \cdot b_{i k} .
$$


On the other hand, if $m-i+3 \leq j \leq m+1$, setting $k=i+j-m-2$, we have $1 \leq k<i$ and

$$
a_{i j} d_{j}=x_{k}^{\beta_{k}} a_{i k}^{\prime} \cdot d_{k-i+m+2}=x_{k}^{\beta_{k}} \cdot b_{i k} .
$$

Thus, we get

$$
\sum_{k=1}^{m+1} b_{i k} \cdot x_{k}^{\beta_{k}}=0
$$

for all $i=1,2, \ldots, m$, which means the required equality.

For any $k=1,2, \ldots, m+1$, we denote by $B_{k}$ the submatrix of $B$ determined by removing the $k$-th column. We set $b_{k}=(-1)^{k-1} \operatorname{det} B_{k} \in$ $I^{m}$.

Proposition 4.4. Suppose $\beta_{k}=\alpha_{k, 1}$ for any $k=1,2, \ldots, m$ (For example, this holds if $\alpha_{k, 1}=1$ for any $\left.k=1,2, \ldots, m\right)$. Then, there exists $\delta \in R$ such that $x_{k}^{\beta_{k}} \cdot \delta=b_{k}$ for any $k=1,2, \ldots, m+1$ and $\left(I^{m}\right)^{\text {sat }}=I^{m}+(\delta)$.

Proof. The existence of $\delta$ such that $x_{k}^{\beta_{k}} \cdot \delta=b_{k}$ for any $k=$ $1,2, \ldots, m+1$ follows from Lemmas 2.3 and 4.3. Then $\delta \in I^{m}:_{R}$ $Q \subseteq\left(I^{m}\right)^{\text {sat }}$. We put $Q^{\prime}=\left(x_{1}^{\beta_{1}}, x_{2}^{\beta_{2}}, \ldots, x_{m}^{\beta_{m}}\right) R$. Then

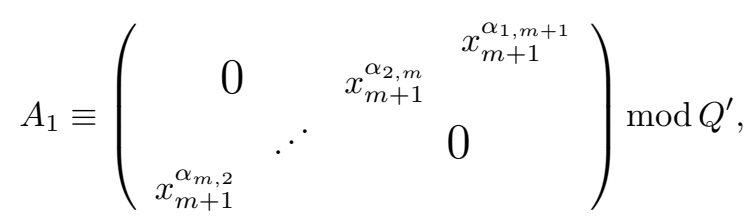

and so $d_{1} \equiv \pm x_{m+1}^{\alpha} \bmod Q^{\prime}$, where $\alpha:=\alpha_{1, m+1}+\alpha_{2, m}+\cdots+\alpha_{m, 2}$. Furthermore, if $2 \leq k \leq m+1$, we have $d_{k} \in Q^{\prime}$ since the entries of the first column of $M_{k}$ are $x_{1}^{\beta_{1}}, x_{2}^{\beta_{2}}, \ldots, x_{m}^{\beta_{m}}$. Hence $Q^{\prime}+I=Q^{\prime}+\left(x_{m+1}^{\alpha}\right)$. On the other hand, the assumption of Proposition 4.4 implies that, for all $k=1,2, \ldots, m$, we can take $k$ itself as $i_{k}$, and then $a_{k k}^{\prime}=1$. Hence,

$$
B=\left(\begin{array}{cccccc}
d_{1} & a_{12}^{\prime} d_{2} & a_{13}^{\prime} d_{3} & \cdots & a_{1 m}^{\prime} d_{m} & a_{1, m+1}^{\prime} d_{m+1} \\
a_{21}^{\prime} d_{m+1} & d_{1} & a_{23}^{\prime} d_{2} & \cdots & a_{2 m}^{\prime} d_{m-1} & a_{2, m+1}^{\prime} d_{m} \\
a_{31}^{\prime} d_{m} & a_{32}^{\prime} d_{m+1} & d_{1} & \cdots & a_{3 m}^{\prime} d_{m-2} & a_{3, m+1}^{\prime} d_{m-1} \\
\cdots & \cdots & \cdots & \cdots & \cdots & \cdots \\
a_{m 1}^{\prime} d_{3} & a_{m 2}^{\prime} d_{4} & a_{m 3}^{\prime} d_{5} & \cdots & d_{1} & a_{m, m+1}^{\prime} d_{2}
\end{array}\right),
$$


and so

$$
B_{m+1} \equiv\left(\begin{array}{cccc} 
\pm x_{m+1}^{\alpha} & & & \\
& \pm x_{m+1}^{\alpha} & & 0 \\
& 0 & \ddots & \\
& & & \pm x_{m+1}^{\alpha}
\end{array}\right) \bmod Q^{\prime}
$$

which means $b_{m+1} \equiv \pm x_{m+1}^{m \alpha} \bmod Q^{\prime}$. Thus, we get

$$
x_{m+1}^{\beta_{m+1}} \cdot \delta \equiv x_{m+1}^{m \alpha} \bmod Q^{\prime}
$$

Here we notice $\beta_{m+1} \leq \alpha_{1, m+1}<\alpha$. Because $x_{1}^{\beta_{1}}, x_{2}^{\beta_{2}}, \ldots, x_{m+1}^{\beta_{m+1}}$ is an $R$-regular sequence, it follows that

$$
\delta \equiv \pm x_{m+1}^{m \alpha-\beta_{m+1}} \bmod Q^{\prime}
$$

and so

$$
Q^{\prime}+(\delta)=Q^{\prime}+\left(x_{m+1}^{m \alpha-\beta_{m+1}}\right) \supseteq Q^{\prime}+\left(x_{m+1}^{m \alpha}\right)=Q^{\prime}+I^{m} .
$$

Now we consider the $R$-linear map

$$
f: R \longrightarrow \frac{Q^{\prime}+\left(x_{m+1}^{m \alpha-\beta_{m+1}}\right)}{Q^{\prime}+\left(x_{m+1}^{m \alpha}\right)}=\frac{Q^{\prime}+(\delta)}{Q^{\prime}+I^{m}}
$$

such that $f(1)$ is the class of $x_{m+1}^{m \alpha-\beta_{m+1}}$. Then we have the following:

Claim. Ker $f=Q$.

If this is true, then $R / Q \cong\left(Q^{\prime}+(\delta)\right) /\left(Q^{\prime}+I^{m}\right)$, and so

$$
\ell_{R}(R / Q)=\ell_{R}\left(\frac{Q^{\prime}+(\delta)}{Q^{\prime}+I^{m}}\right)
$$

Because $\left(Q^{\prime}+(\delta)\right) /\left(Q^{\prime}+I^{m}\right)$ is a homomorphic image of $\left(I^{m}+(\delta)\right) / I^{m}$ and $I^{m}+(\delta) \subseteq\left(I^{m}\right)^{\text {sat }}$, we have

$$
\ell_{R}\left(\frac{Q^{\prime}+(\delta)}{Q^{\prime}+I^{m}}\right) \leq \ell_{R}\left(\frac{I^{m}+(\delta)}{I^{m}}\right) \leq \ell_{R}\left(\left(I^{m}\right)^{\mathrm{sat}} / I^{m}\right)=\ell_{R}(R / Q),
$$

where the last equality follows from Proposition 4.2. Thus, we see

$$
\ell_{R}\left(\frac{I^{m}+(\delta)}{I^{m}}\right)=\ell_{R}\left(\left(I^{m}\right)^{\mathrm{sat}} / I^{m}\right)
$$


and so $I^{m}+(\delta)=\left(I^{m}\right)^{\text {sat }}$ holds.

Proof of Claim. Let us take any $z \in \operatorname{Ker} f$. Then, there exists $w \in R$ such that

$$
z \cdot x_{m+1}^{m \alpha-\beta_{m+1}} \equiv w \cdot x_{m+1}^{m \alpha} \bmod Q^{\prime} .
$$

This congruence implies

$$
x_{m+1}^{m \alpha-\beta_{m+1}}\left(z-w \cdot x_{m+1}^{\beta_{m+1}}\right) \in Q^{\prime} .
$$

Because $x_{1}^{\beta_{1}}, \ldots, x_{m}^{\beta_{m}}, x_{m+1}^{m \alpha-\beta_{m+1}}$ is an $R$-regular sequence, we have $z-w \cdot x_{m+1}^{\beta_{m+1}} \in Q^{\prime}$, which means $z \in Q$. Hence, Ker $f \subseteq Q$. As the converse inclusion is obvious, we get the equality of the claim, and the proof of Proposition 4.4 is complete.

\section{REFERENCES}

1. K. Akin, D. Buchsbaum and J. Weyman, Resolutions of determinantal ideals: the submaximal minors, Adv. Math. 39 (1981), 1-30.

2. L. Avramov, Complete intersections and symmetric algebras, J. Alg. 73 (1981), 248-263.

3. W. Bruns, A. Conca and M. Varbaro, Maximal minors and linear powers, preprint (2013), arXive:1203.1776.

4. W. Bruns and J. Herzog, Cohen-Macaulay rings, Cambr. Stud. Adv. Math. 39, Cambridge University Press, 1997.

5. S.D. Cutkosky, Symbolic algebras of monomial primes, J. reine angew. Math. 416 (1991), 71-89.

6. K. Fukumuro, On the symmetric and Rees algebras of certain determinantal ideals, Tokyo J. Math., to appear.

7. K. Fukumuro, T. Inagawa and K. Nishida, On a transform of an acyclic complex of length 3, J. Alg. 384 (2013), 84-109.

8. S. Goto, K. Nishida and Y. Shimoda, The Gorensteinness of symbolic Rees algebras for space curves, J. Math. Soc. Japan 43 (1991), 465-481.

9. mial curves, Trans. Amer. Math. Soc. 340 (1993), 323-335.

10. , Topics on symbolic Rees algebras for space monomial curves, Nagoya Math. J. 124 (1991), 99-132.

11. S. Goto, K. Nishida and K. Watanabe, Non-Cohen-Macaulay symbolic blowups for space monomial curves and counterexamples to Cowsik's question, Proc. Amer. J. Math. 120 (1994), 383-392.

12. J. Herzog, Generators and relations of abelian semigroups and semigroup rings, Manuscr. Math. 3 (1970), 175-193. 
13. C. Huneke, Hilbert functions and symbolic powers, Michigan Math. J. 34 (1987), 293-318.

14. T. Inagawa, *-transforms of acyclic complexes, preprint (2013), arXive: 1307.1500 .

15. G. Knödel, P. Shenzel and R. Zonsarow, Explicit computations on symbolic powers of monomial curves in affine space, Comm. Algebra 20 (1992), 2113-2126.

16. K. Kurano and N. Matsuoka, On finite generation of symbolic Rees rings of space monomial curves and existence of negative curves, J. Alg. 322 (2009), 3268-3290.

17. M. Morales and A. Simis, Arithmetically Cohen-Macaulay monomial curves in $\mathbb{P}^{3}$, Comm. Alg. 21 (1993) 951-961.

18. L. O'Carroll and F. Planas-Vilanova, Ideals of Herzog-Northcott type. Proc. Edinb. Math. Soc. 54 (2011), 161-186.

19. M.E. Reed, Generation of symbolic blow-ups of monomial space curves in degree four, Comm. Alg. 33 (2005), 2541-2555.

20. , Generation in degree four of symbolic blowups of self-linked monomial space curves, Comm. Alg. 37 (2009), 4346-4365.

21. H. Srinivasan, On finite generation of symbolic algebras of monomial primes, Comm. Alg. 19 (1991), 2557-2564.

Graduate School of Science, Chiba University, 1-33 Yayoi-Cho, Inage-Ku, CHIBA-ShI, 263-8522, JAPAN

Email address: blackbox@tempo.ocn.ne.jp

Graduate School of Science, Chiba University, 1-33 Yayoi-Cho, Inage-Ku, ChibA-Shi, 263-8522, JAPAN

Email address: kado@ta3.so-net.ne.jp

Institute of Management and Information Technologies, Chiba University, 1-33 Yayoi-Cho, Inage-Ku, Chiba-Shi, 263-8522, Japan

Email address: nishida@math.s.chiba-u.ac.jp 